\section{Integrating High Resolution Light Microscopy and Real Time Observation of Fluorescent Labels}

\author{
Thomas A. Hasling \\ Aetos Technologies, Inc., Auburn, AL \\ tom.hasling@aetostech.com
}

Fluorescence microscopy has experienced tremendous growth over the past four decades and has facilitated major advancements in science. The classic fluorescent techniques (which include epifluorescence and confocal) allow researchers to selectively observe labeled structures with great clarity and consistency. Historically, biological scientists have been the most prolific users of fluorescence imaging. However, growing numbers of nano-materials research initiatives are now incorporating quantum dots and other fluorescent labels into their imaging protocols. This is especially true in areas where nano-materials and biological research is overlapping such as drug delivery. This nano-bio convergence, along with other advancements, has generated the need to observe highly dynamic events involving labeled and unlabeled structures in real time.

For many researchers, observing the sample portion that is fluorescently labeled is often not sufficient. Many times it is important to visualize the fluorophore labeled components in the full context of the sample. Traditional widefield epi-fluorescence and confocal microscopy are highly effective techniques for quantifying that portion of the sample that is fluorescent. However, to visualize these fluorescent components in the full context of the sample, additional imaging techniques, such as differential interference contrast (DIC) and phase contrast are often utilized. Traditionally, these techniques are used to capture the refractive structure of the sample. These images are then computationally combined with the fluorescent image in what is commonly referred to as an "overlay." While this overlay technique is sufficient for some applications, it precludes the observation of real time interactions between fluorescent and non-fluorescent portions of the sample. For example, in nano-drug delivery, the ability to track cellular uptake of fluorescent nano-particles while observing consequences such as cell morphological changes and transport mechanisms may provide insights previously unavailable. To capture these dynamic processes

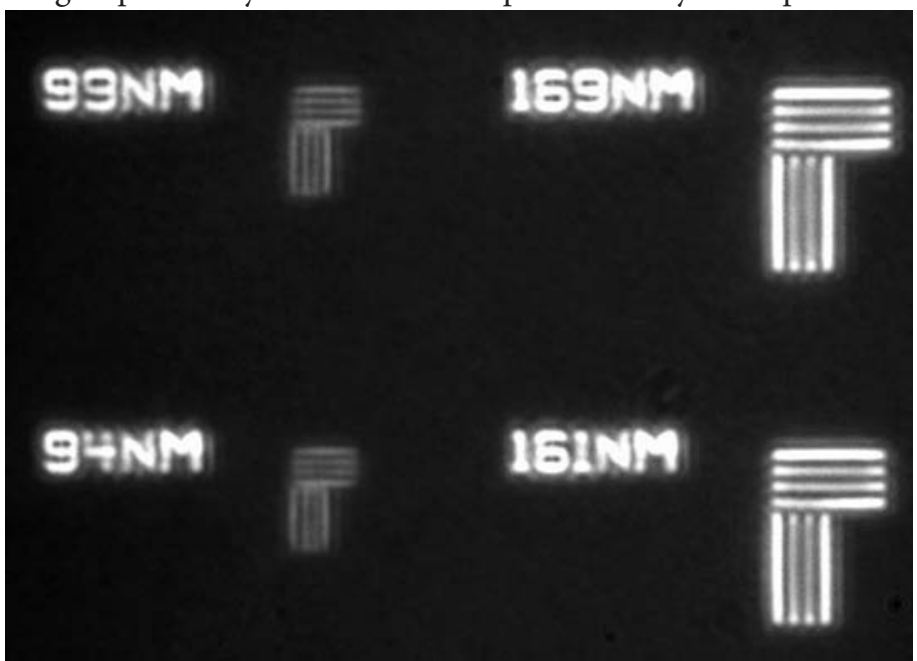

Figure 1 is an imaged portion of the Richardson Test Slide Gen III. The vertical and horizontal lines are separated by distance indicated by the label. Imaged using CytoViva, Olympus BX-51, 100x oil objective NA 1.4, Sony DXC-S500color digital camera.

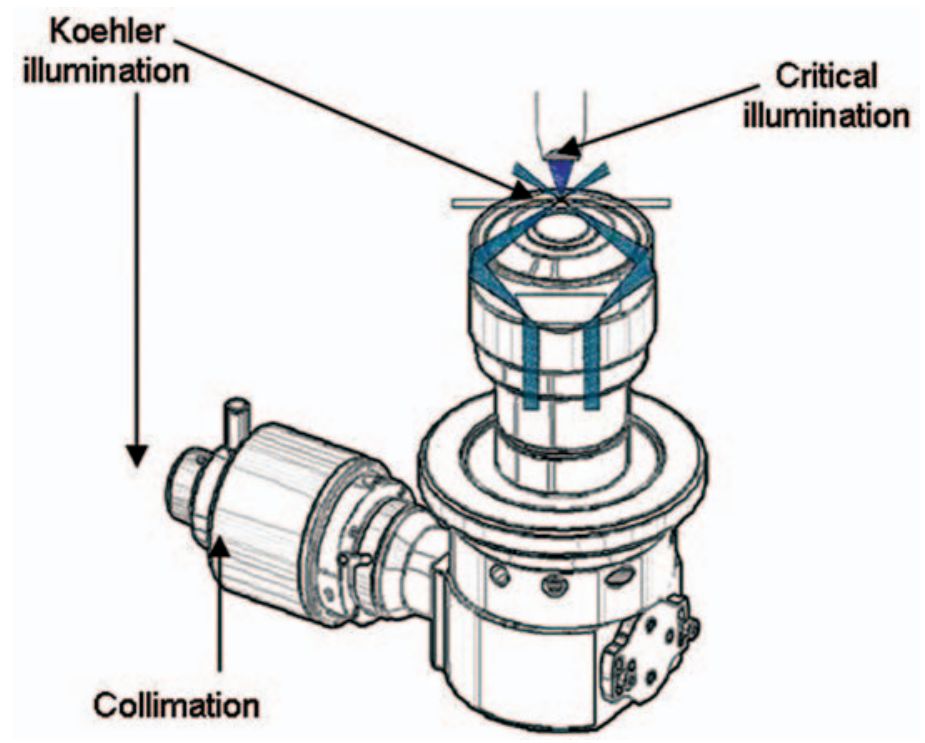

Figure 2 illustrates the basic light path of the CytoViva high resolution adapter.

in real time, it is very useful to have a microscopy technique that allows direct, simultaneous observation of both the fluorescent and non-fluorescent sample portions.

This ability to observe both fluorescent and non-fluorescent components simultaneously is available utilizing the CytoViva ${ }^{\mathrm{TM}}$ high resolution, Dual Mode Fluorescence (DMF) system. Originally introduced as a tool to produce superior optical images of cellular structures and nano-materials, the high resolution adapter replaces the condenser on a standard research grade microscope. This system incorporates fixed Koehler Illumination and a highly efficient hollow cone of light to illuminate the sample. In doing so, it narrows the point-spread function of the incident light. These attributes provide illumination that allows sub $100 \mathrm{~nm}$ resolution to be achieved when combined with a high N/A oil objective [1] (see figure 1). This allows observation of small intracellular bacteria or nano-particles, which are often too small to be seen with DIC, brightfield and other common optical illumination techniques. Additionally, the pre-aligned, fixed geometry of the system enables

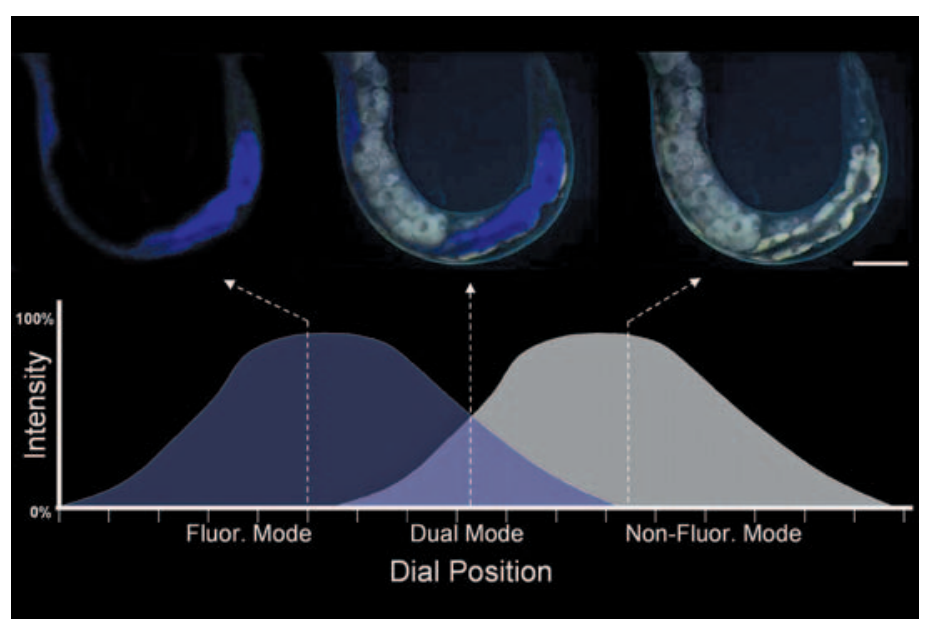

Figure 3 depicts three representative images of a C. elegan with autofluorescent bacteria (blue) in the gut. Three distinct modes are represented: fluorescence only, dual mode fluorescence and high resolution, nonfluorescent mode. The graph indicates the relative mixture of illumination selected by the DMF module and indicates the image produced. Imaged using CytoViva/DMF, Olympus BX-51, $20 x$ objective NA .6, Hitachi KPD20B 1/2" digital video camera (50 um scale bar). 
The State of the Art, Revolutionary

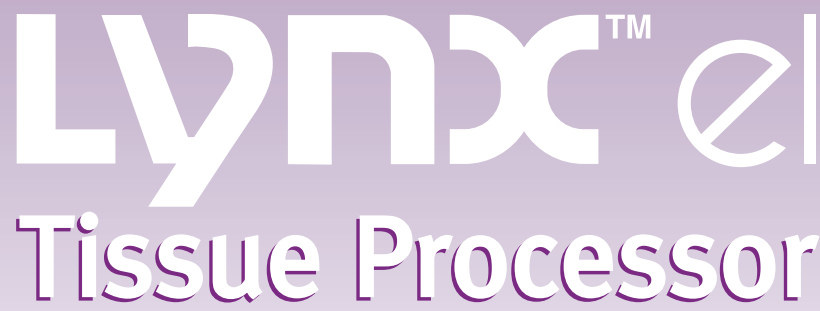

The EMS LMNX Automated Tissue Processor now made even better... for Electron Microscopy and Light Microscopy.

Dramatically reduce your sample preparation cost and time with improved reproducible results!!

The EMS LMN allows for the processing of tissue all the way through $100 \%$ resin.

\section{Primary uses:}

- Ultrastructural Studies

- Extremely Hard Tissues - Undecalcified Bone

- High Resolution Light Microscopy

- Immunohistochemistry

- Dehydration for SEM

- Deparaffinization

- En-bloc Staining

- And Much More

This state of the art Automated Tissue Processor has many unique features making the unit the most versatile and reliable unit on the market at a very competitive price.

\section{Features:}

- Stainless Steel Working Surfaces

- Enclosed Benchtop Unit

- Reagent Turntable/ Max of 56 Specimens

- 20 Reagent Vials/Cycle

- Temp Range of $4^{\circ} \mathrm{C}-60^{\circ} \mathrm{C}$

- Built in Exhaust Fan

- Alarm Monitoring System

- Multiple Safety Features

- Battery Back Up

- Storage of 10 Programs/ Each with 20 Pre-programmable Steps

- Printer Output

- Delay Start of Programs

- And Much More...

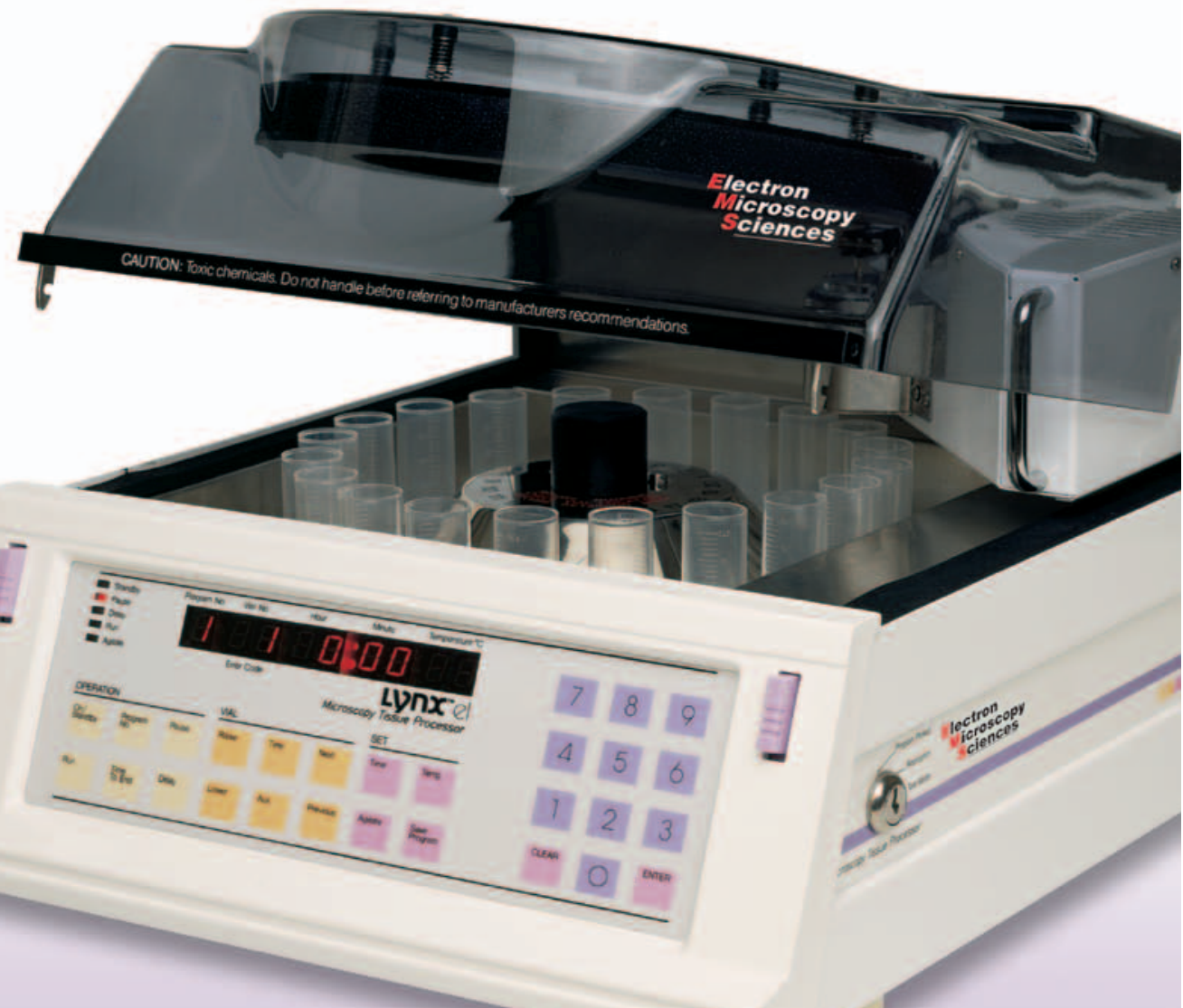

For more information or a complete brochure on the "LMN" Automated Tissue Processor, please call or write us today. 


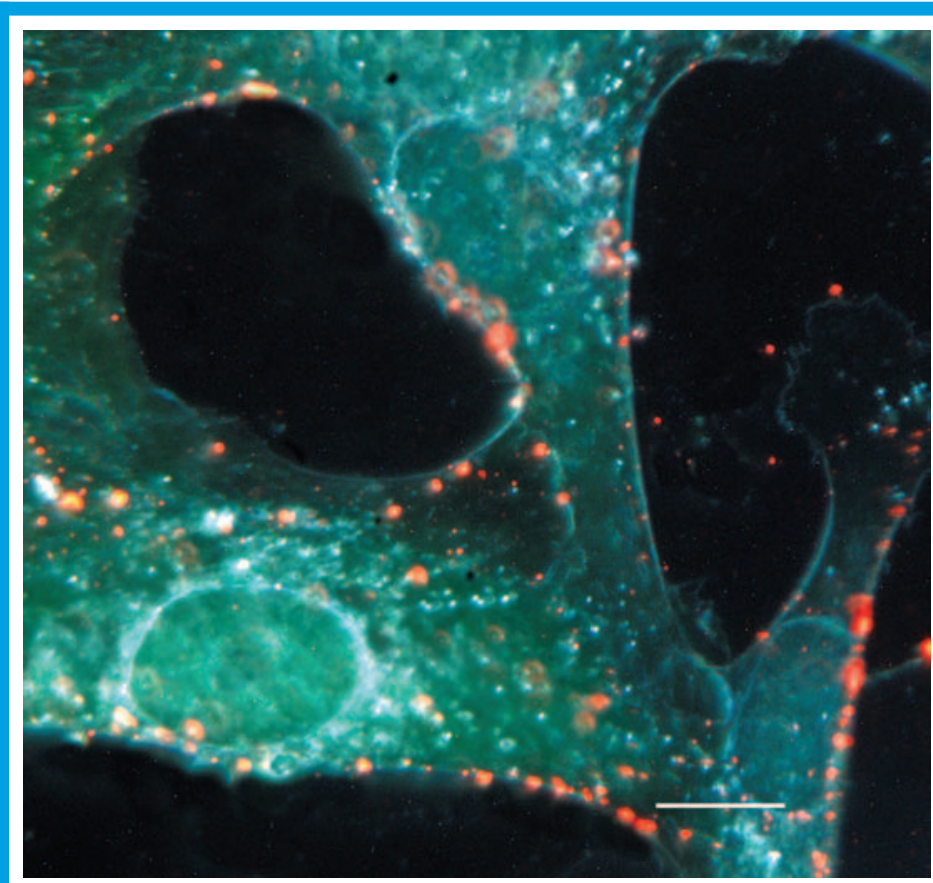

Figure 4 depicts Rhodamine labeled 200nm liposomes interacting with the membrane of live cultured fibroblasts. Imaged using CytoVival DMF, Olympus BX-51, 40x objective NA 1.0, Optronics Micro-fire color digital camera (20 $\mu \mathrm{m}$ scale bar).

broader use by researchers not trained in more complex and time consuming microscopy techniques (see figure 2).

The Dual Mode Fluorescence (DMF) module was recently added to the high resolution capability of CytoViva. DMF is a transmitted light fluorescence imaging technique that allows both fluorescently labeled and non-labeled sample structures to be observed simultaneously and in real time. The DMF system utilizes a series of excitation filters and other optical components that allow the user to precisely control the mixture of excitation energy and white light. The system is configured in a unique and proprietary manner that allows light passing through the optical path to be easily modulated between: 1) fluorescence only imaging 2) simultaneous fluorescence and non-fluorescence imaging or 3) high resolution, non-fluorescent imaging (see figure 3). Above the objective, DMF utilizes a specialized filter that allows selected emission peaks to be passed. Additionally, the transmission peaks will recombine to form a wide spectrum image allowing the observation of both the fluorescent and refractive portions of the sample. This emission filter can be mounted in any available filter cube or filter tray above the objective. The excitation band is selected via the DMF module. Here, for example, one can select green, independent of red, or conduct multi-pass excitation. In both instances you can also observe the non-labeled structures simultaneously with the selected excitation. Using multiple filter configurations, a wide variety of fluorophores can be observed with this technique. This can include specialized filter sets optimized for quantum dot observation. Both excitation and emission filters used in this technique are available from major filter manufacturers. The DMF technique uses a classic mercury halide illumination source with spectral peaks optimized for fluorescence. The light source, DMF module and high resolution adapter are connected via liquid light guides. This modular configuration allows the technique to be utilized on a wide range of upright and inverted light microscopes.

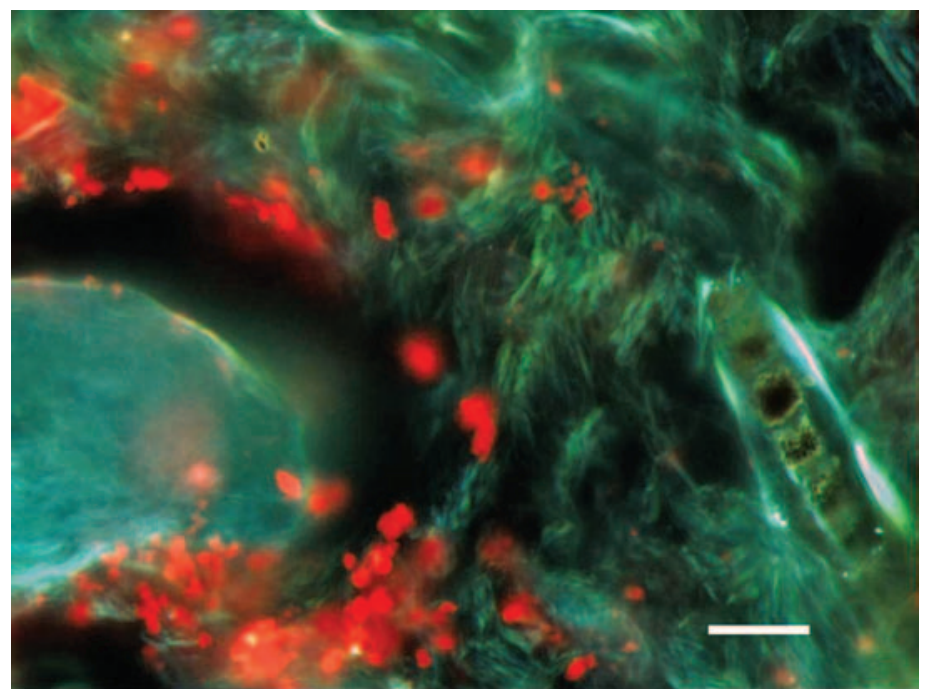

Figure 5 depicts fluorescently labeled silica nano-particles interacting with mouse skin tissue. Imaged using CytoViva/DMF, Olympus BX-51, 40x objective NA 1.0, Optronics Micro-fire color digital camera (100 $\mu \mathrm{m}$ scale bar). Sample provided by Dr. Steve Roberts University of Florida.

\section{Nano-Bio Applications}

The convergence of nano-materials and biological sciences has become significant for both academic and industrial research communities. Applications that utilize nano-materials to manipulate and interact with biological specimens hold great promise. It also represents one of the fastest growing segments of basic research. This is evidenced by federal government funding initiatives in this area that have more than doubled to over $\$ 1$ billion since 2001 [2]. Nano-bio related research is also driving the need for new fluorescence microscopy techniques. Examples of such research include the utilization of nano-particles as delivery agents for cancer therapeutics and other drugs. Recently, efforts to utilize nano-sized liposomes as drug carriers have gained significant popularity due to characteristics such as solubilisation, targeting options, and cellular internalization [3]. Figure 4 illustrates the ability of the DMF technique to observe and record (in real time) the interactions between

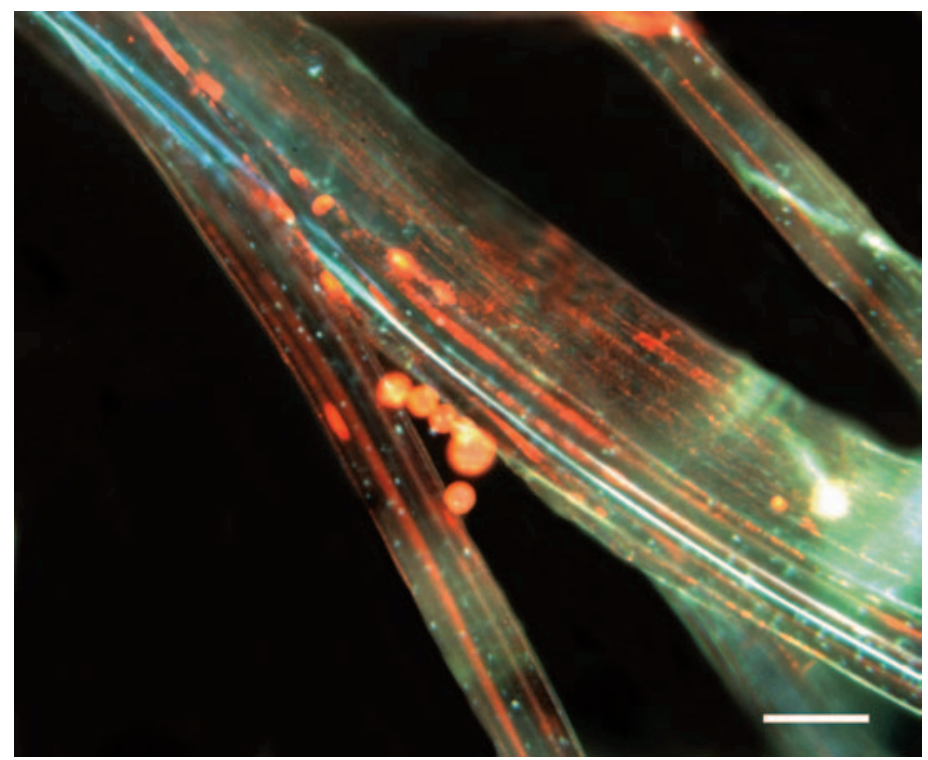

Figure 6 depicts polyester fibers and with adherent quantum dots. Imaged using CytoViva/DMF, Olympus BX-51 40x objective NA .6, Optronics Micro-fire color digital camera (50 $\mu \mathrm{m}$ scale bar). 


\section{Soft Imaging System}

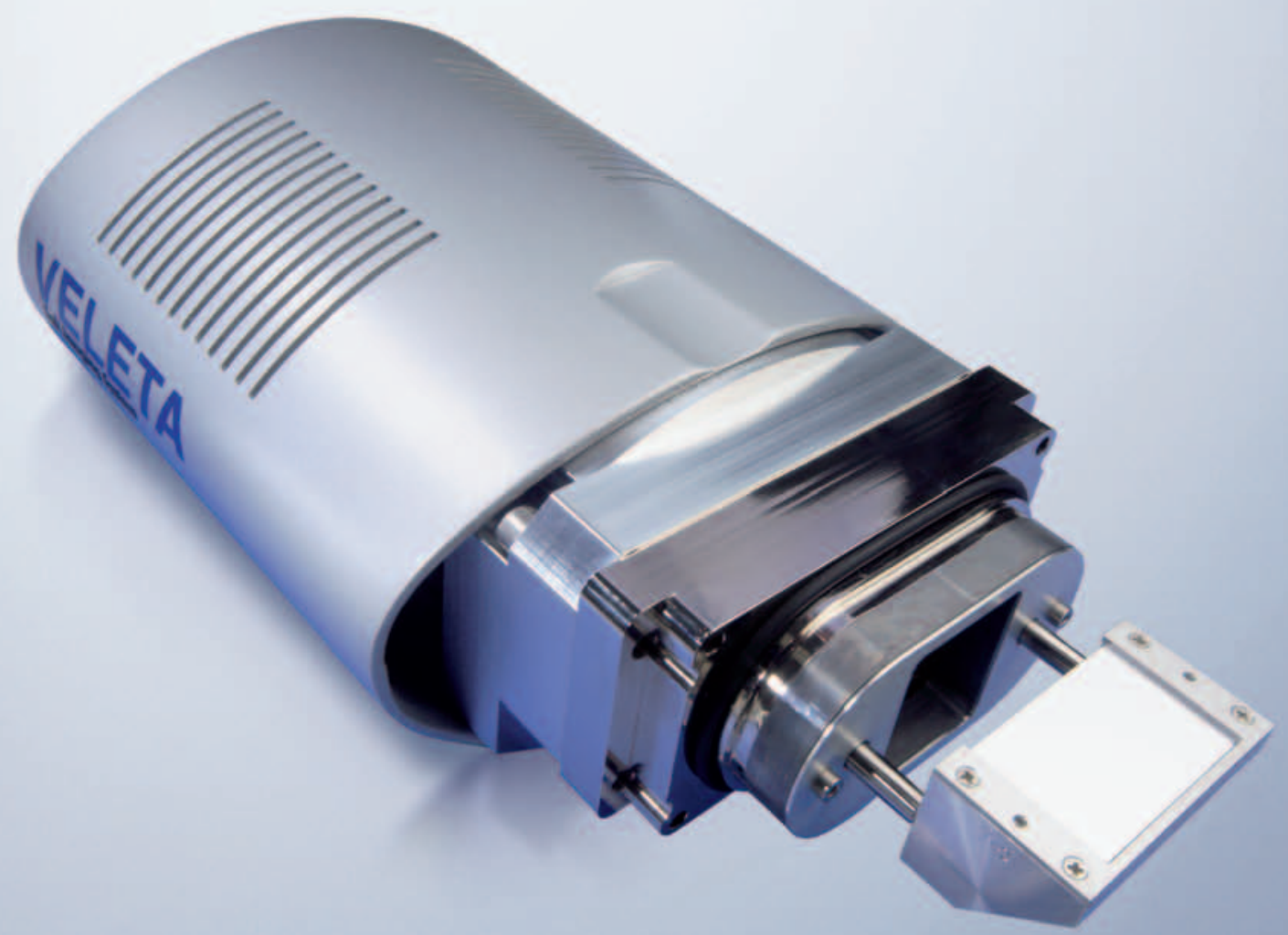

\section{YOUR SPECIFICATIONS - OUR SOLUTION VELETA - 2k x 2k SIDE-MOUNTED TEM CAMERA SOLUTION}

Meeting the demands on today's TEM camera market - our new side-mounted TEM camera has the capabilities that customers are looking for in a $2 \mathrm{k} \times 2 \mathrm{k}$ camera. The new Veleta fills this description perfectly. If you're familiar with our MegaView III and Morada cameras, the Veleta's capabilities position it right between these two.

This new side-mounted TEM camera is our answer to the most current expectations on the TEM market. The Veleta is a Peltier-cooled camera featuring up to $2048 \times 2048$ highly sensitive pixels and has a 14-bit dynamic range. Veleta offers more than $10 \mathrm{fps}$ at full view and about $20 \mathrm{fps}$ at binning mode 2.

Veleta is optimized for today's latest applications - such as tomography or immunogold labeling. Naturally the camera is applicable for all standard TEM imaging tasks.

TEM - Cameras
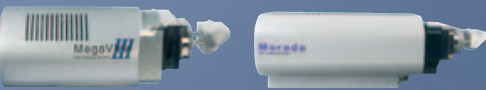

For detailed information please contact:

Olympus Soft Imaging Solutions

info.osis@olympus-sis.com

www.soft-imaging.net 


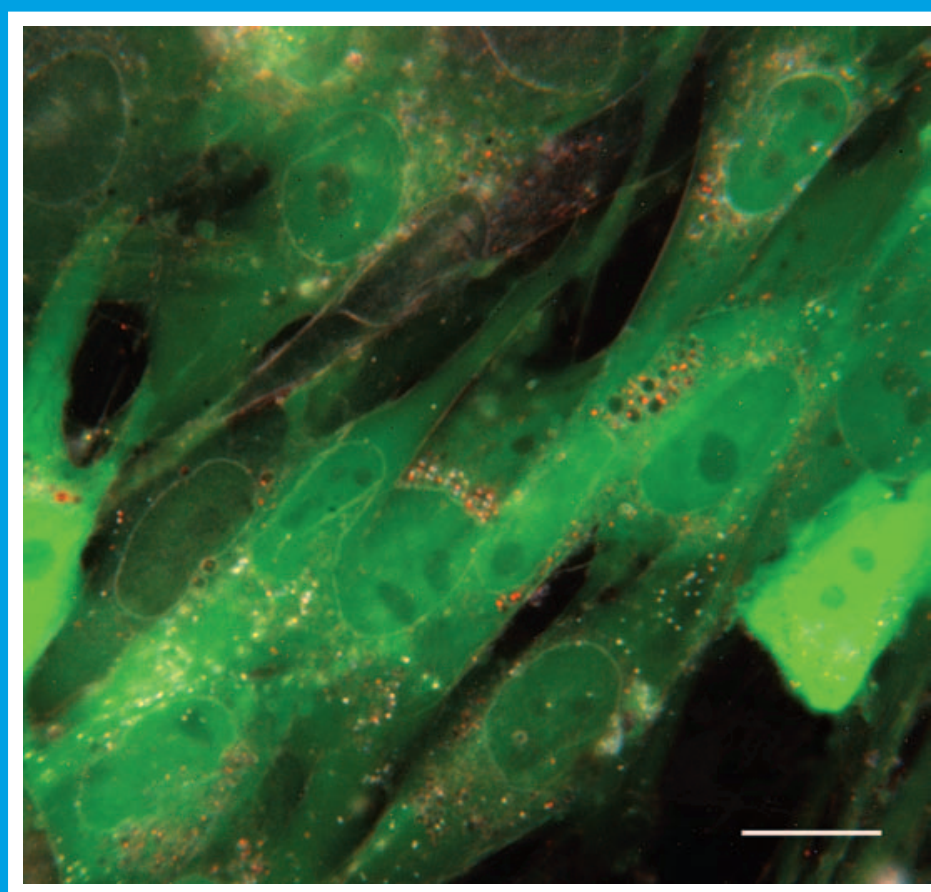

Figure 7 depicts live cultured mesenchymal stromal cells (MSC) transfected with a green fluorescent protein. Note: the MSCs were cultured with and have internalized red quantum dots, which can be seen within vesicles in the granular zones. Imaged using CytoViva/DMF, Olympus BX$5140 x$ objective NA .6, Optronics Micro-fire color digital camera $(20 \mu \mathrm{m}$ scale bar). Sample provided by Dr. Doug Martin, Scott-Ritchey Research Institute, Auburn University.

fluorescently labeled liposomes and live unlabeled fibroblasts. Note in this image how the liposomes can be observed interacting with the cell membrane.

Nano-materials have already moved out of the lab and are being incorporated into our everyday lives. For example, silver nano-particles have been integrated into clothing and other materials due to their anti-microbial properties. The promise held by these advancements has led to increased efforts through the National Nanotechnology Initiative to study the potential effects of nano-materials on both humans and the environment [4]. For experiments that quantify the toxicity of certain nano-materials, it is useful to observe and record in real time the interactions between these materials and other specimens such as skin tissue. Figure 5 illustrates such an experiment by demonstrating the interaction between fluorescent silica nano-particles and mouse skin tissue. The ability to capture both the fluorescent particles and tissue structure simultaneously allows the researcher to understand particle location and its effect on the surrounding tissue.

\section{Materials Applications}

For scientists involved in nano-materials development, light microscopy is often viewed as a complimentary tool to SEM, TEM and AFM systems. For example, research conducted by NASA recently noted that optical microscopy is an important tool for initial assessments of single wall nanotubes (SWNT) to evaluate dispersions of aggregates before moving on to higher resolution microscopy methods. As such, high contrast optical imaging methods that maximize resolution are fast becoming an important tool supporting research efforts in the rapidly growing field of carbon nanotube research [5]. For certain types of materials, light microscopy can also provide new information regarding materials-based samples that cannot be gained through electron microscopy. For example, high resolution light microscopy provides the ability to observe structural details in a wide range of emulsion and polymer based samples as shown in Figure 6. Finally, high contrast, high resolution optical microscopy can also serve as a fast, easy to use, quality control tool for the evaluation of particle dispersion and agglomeration in composite materials such as thin films [6].

\section{Biological Applications}

Biological sciences have long been the primary domain of fluorescence-based microscopy due to the high level of specificity available through immunocytochemistry. However the growing need to observe live cells, combined with an ever-expanding number of fluorescent reagents and transgenic fluorescent proteins increases the demand for a wide range of fluorescent imaging techniques.

Epi-fluorescent and confocal imaging techniques provide effective observation of fluorescent labels. However, the independent observation of the DIC or phase contrast as an overlay image is consequentially non-real time. The use of CytoViva DMF allows the researcher to maximize the advantages of many of the physiologic reagents and modern biological fluorescent techniques by allowing for real time observation of fluorescence in the full context of the sample environment. Not only does this technique provide real time context of the fluorophore labels within the sample, interactions between these sample components can also be easily observed (see figure 7). This is a fast and efficient process for identifying those sample elements of interest. It also allows the image to be quickly analyzed for counts of fluorescent versus non-fluorescent elements.

CytoViva's ability to resolve below $100 \mathrm{~nm}$ coupled with real time simultaneous observation of both fluorescent and non-fluorescent sample structures has ubiquitous appeal in both the biological and materials sciences. Separating this technique from others is the efficient and straightforward manner in which a researcher can directly observe and capture images without the need of computationally manipulating multiple images. The technique can also solve a number of practical problems encountered by fluorescent microscopists. For example, researchers scanning for labels across large areas often do not know if they are in the proper focal plane or if the label is associated with the structure of interest. DMF allows this researcher to easily transition between the modes (figure 3 ) and determine if the field is relevant.

Indeed, this powerful and accessible technique combines two unique properties that serve to benefit a wide range of research interests including the rapid evolution of biological science and its convergence with nanotechnology.

\section{References}

[1] Resolution of $90 \mathrm{~nm}(\lambda / 5)$ in an optical transmission microscope with an annular condenser, Optics Letters, Volume 31 Issue 19, October 2006.

[2] Nanotech Facts National Nanotechnology Initiative www.nano.gov/ html/faqs.html

[3] Liposomes - Successful Carrier Systems for Targeted Drug Delivery Utrecht Institute for Pharmaceutical Sciences http://www.samedanltd. com

[4] National Toxicology Program National Nanotechnology Initiative www. nano.gov/html/society/EHS2.htm

[5] NIST Practice Guide: Optical, Electron and Probe Microscopy Characterization of Carbon Nanotube Composites.

[6] Nanoscience Group University of Nottingham www.nottingham. ac/physics/research/nano/optmicro.htm 


\section{CUSTOMIZING \\ TOYOUR SPECIFIC NEEDS}

Micro-manipulators, preparation materials, darkroom and general lab supplies, books, grids and apertures. Many items are manufactured in our machine shop, so customizing to your specific need is not a problem.

Some of the accessories and laboratory supplies we can supply are tweezers, tools, TEM CCD imaging systems, tensile testers, turbo evaporators, sputter coaters, substages, specimen holders, standards, carbon coaters, and more...
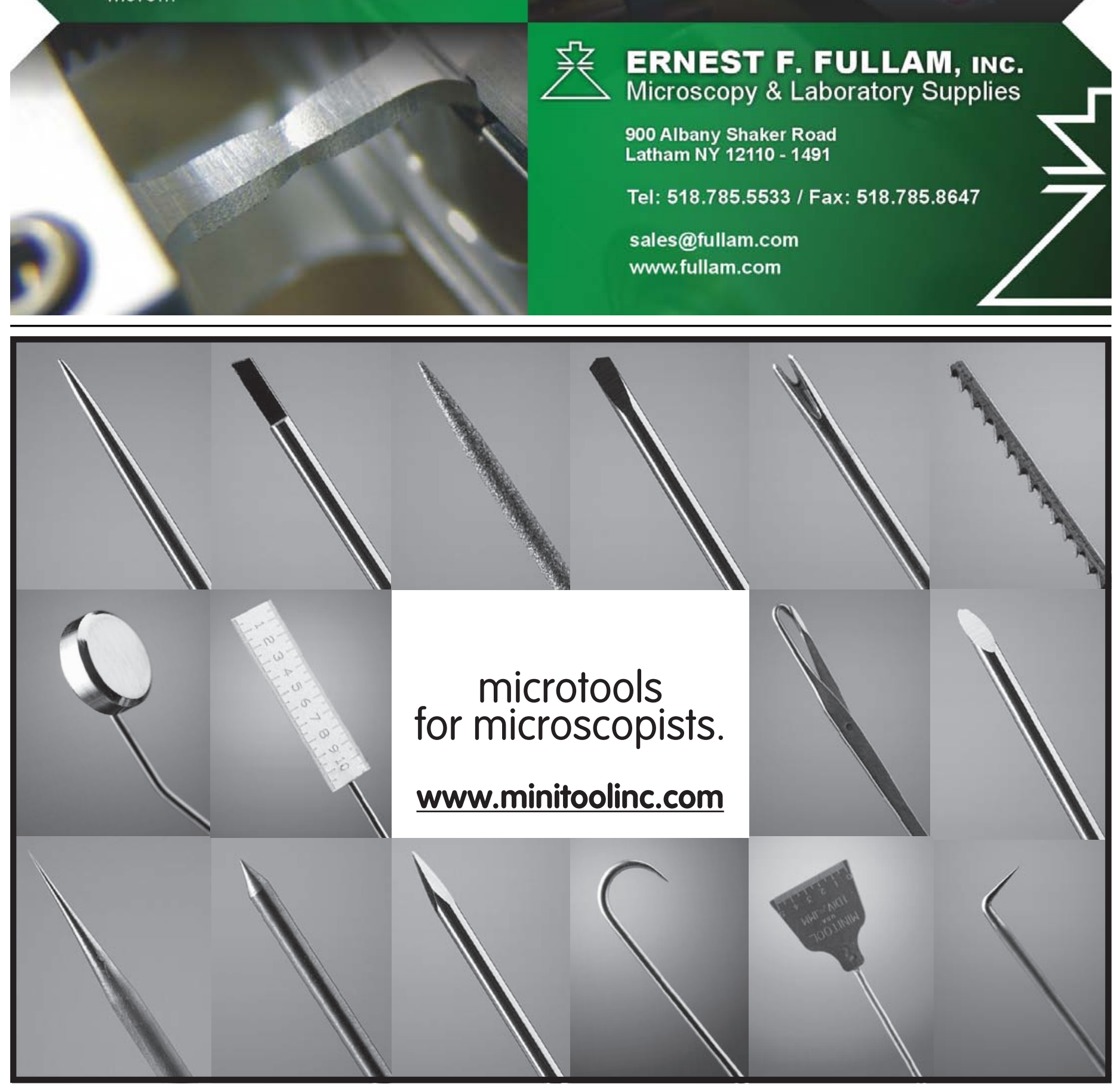